\title{
SOCIODEMOGRAPHIC, CLINICO-LABORATORY CHARACTERISTICS WITH SHORT OUTCOME AMONG ACUTE HEART FAILURE PATIENTS ADMITTED FOR FIRST TIME IN A TERTIARY CARE HOSPITAL: A REAL LIFE EXPERIENCE
}

\author{
KABIR AKMH ${ }^{1}$, SAYEED SKJB ${ }^{2}$, SARKAR PK ${ }^{3}$, HOSSAIN MZ ${ }^{4}$, RAHMAN ${ }^{5}$, RANA MM ${ }^{6}$, \\ MAJUMDER MS ${ }^{7}$, BASAK DK ${ }^{8}, \mathrm{KABIR} \mathrm{ABMS}^{9}$, ADNAN MA $^{10}$
}

\begin{abstract}
Acute heart failure is one of the common causes of morbidity \& mortality among the patients admitted in hospital. This prospective observational study was done to characterize sociodemographic, clinical \& laboratory characteristics with short clinical outcome. Total 110 patients of acute heart failure were included in this study where mean age was 53.88 ( \pm 14.6$)$ years. Majority of the patients were aged between 51-60 years 35 (32\%). 79(72\%) of them smoker and 12(11\%) were alcoholic. Major comorbidities were hypertension 59 (53.63\%), ischemic heart disease 42 (38.18\%), DM 39(35.45\%), chronic kidney disease 25(22.72\%). Most common symptoms at presentation were shortness of breath110(100\%) along with palpitation 96(87.27\%), orthopnea 85(77.27\%), paroxysmal nocturnal dyspnea 59(53.63\%), ankle edema 71(64.54\%), raised JVP $75(68.18 \%)$, bilateral basal crepitation on auscultation 80(72.72\%), anemia 19 (17.27\%) \& oliguria 23(20.90\%) where mean duration of symptoms was $11 \pm 2$ days. Lab investigations revealed decreased Hemoglobin (10.71 \pm 1.49$) \mathrm{g} / \mathrm{dl}$, proteinuria in 31 (28.18\%), ketone body 5 (4.5\%), raised creatinine in 23(20.93\%), hyponatremia 38 (34.54\%), hypernatremia 6 (5.45\%), hypokalemia 6(5.45\%), hyperkalemia 3 (2.72\%). Regarding cardiac events 37(33.63\%) encounter myocardial infarction, cardiogenic shock 29(26.36\%), Cardiac arrhythmia 27 (24.5\%), cardiac arrest 6 (5.45\%), non-cardiac events like stroke 6(5.45\%), hyperosmolar hyperglycemic state 2(1.8\%), diabetic ketoacidosis 5(5.45\%), acute kidney injury 23(20.90\%). However, among cardiac arrhythmia atrial fibrillation found 13(11.81\%) followed by multiple ventricular ectopic 12 (10.90\%), ventricular tachycardia 7 (6.36\%), Complete heart block 6 (5.45\%), variable heart block 4 (3.63\%), long QT 2(1.8\%). Regarding short clinical outcome 52(47.27\%) were improved, 40 (36.36\%) had persistent symptoms \& rest 18 (16.36\%) expired. Most common causes of those deceased person were cardiogenic shock 9(50\%) followed by cardiac arrest 6 (6.33\%). In conclusion we can say clinical characteristics are more or less similar but those who have reduced ejection fraction with comorbidities are very prone to develop cardiac arrhythmia causing sudden cardiac death in heart failure.
\end{abstract}

Key Words: Heart failure, Cardiac arrhythmia, electrolyte imbalance.

J Dhaka Med Coll. 2019; 28(2) : 192-198

\section{Introduction:}

Heart failure $(\mathrm{HF})$ is a leading cause of morbidity and mortality and causes high health care related costs, posing a great burden on both patient and society and accounts for more than 1 million primary and about 3 million secondary annual hospital admissions in the USA alone and it is the most common hospital discharge diagnosis among subjects older than 65 years. The incidence and prevalence of HF rise steeply with age in those aged over 60 years. The most often mentioned prevalence estimate for the

1. Dr. A.K.M. Humayon Kabir, Associate Professor, Dept. of Medicine, Dhaka Medical College Hospital, Dhaka.

2. Dr. S.K. Jakaria Been Sayeed, Indoor Medical Officer, Dept. of Medicine, Dhaka Medical College Hospital, Dhaka.

3. Dr. Prodip Kumar Biswas, Associate Professor, Dept. of Medicine, Dhaka Medical College Hospital, Dhaka.

4. Dr. Mohammad Zaid Hossain, Associate Professor, Dept. of Medicine, Dhaka Medical College Hospital, Dhaka.

5. Dr. Sabrina Rahman, Post graduate trainee, Dept. of Medicine, Dhaka Medical College Hospital, Dhaka.

6. Dr. Md. Masud Rana, Post graduate trainee, Dept. of Medicine, Dhaka Medical College Hospital, Dhaka.

7. Dr. Manash Shekhar Majumder, Post graduate trainee, Dept. of Medicine, Dhaka Medical College Hospital, Dhaka.

8. Dr. Deepankar Kumar Basak, Consultant, Square Hospital, Dhaka

9. ABM Shafiullah Kabir, Consultant, Internal Medicine, Dhaka

10. Dr. Mirza Asif Adnan Assistant Registrar, Dept. of Medicine, Dhaka Medical College Hospital, Dhaka.

Correspondence : Dr. A.K.M. Humayon Kabir, Associate Professor, Dept. of Medicine, Dhaka Medical College Hospital, Dhaka. E-mail-drakmhkabir@gmail.com. Cell no: +8801711137528 
adult population at large is $2 \%(1-3 \%)$, and $5-$ $9 \%$ selectively in those aged 65 years and over. ${ }^{1-}$

${ }^{3}$ Subjects with congestive heart failure $(\mathrm{CHF})$ usually show acid-base and electrolyte disorders, due both to the activation of several neuro-hormonal mechanisms and to drugs used in this condition, such as diuretics. These abnormalities reflect the severity of heart failure and contribute to the functional impairment and to the poor long-term prognosis. 4

The common electrolyte abnormalities are hyponatremia, hypokalemia, and hypomagnesemia. Hypercalcemia and hypomagnesemia also are observed in patients with diabetes, hypertension, and obesity. ${ }^{5}$ Patients with cardiac insufficiency are often administered diuretic therapy that may further exacerbate ion imbalances. ${ }^{6}$ The acid-base disturbances generally observed are metabolic alkalosis pure or combined with respiratory alkalosis. ${ }^{4}$ Several mechanisms interact to produce these alterations. The decrease in cardiac output leads directly to a reduction in renal blood flow, with impairment of renal excretion of water and electrolytes, and it causes the activation of several neurohormonal responses that affect both cardiovascular homeostasis and electrolyte balance. The therapy of $\mathrm{CHF}$ subjects includes the discovery and management of these electrolyte abnormalities that have a role in the development of ventricular arrhythmias. ${ }^{4}$

HF often coexists with a number of prognosisrelevant comorbidities and has direct consequences on other organs, including the kidneys. The relationship of renal function with congestive heart failure (CHF), as highlighted by type I and type II cardio renal syndromes (CRS), is present in both chronic heart failure syndromes as well as acute decompensated heart failure (ADHF). Increasing recognition of the syndrome has revealed a growing incidence of Type I and II as well as the prognostic importance of each, i.e. renal dysfunction in the setting of congestive heart failure portends an independently worse outcome compared to those with preserved renal function. ${ }^{7}$ Progression of HF or kidney disease can have deleterious effects on patient outcomes through the activation of vicious cycles that often accelerate cardiac and renal deterioration. Thus, the heart and kidneys interact in a complex and interdependent manner in both acute and chronic conditions, which can lead to dual organ dysfunction. ${ }^{8,} 9$

The highest risk group for rapid progression of renal dysfunction was the individuals with an eGFR> $90 \mathrm{ml} / \mathrm{min}$ at baseline. ${ }^{10}$ So, it appears that preserved renal function does not protect an individual with systolic dysfunction from developing worsening renal function and those that have renal dysfunction have a poorer prognosis than those with stable, preserved renal function.

Weiner, et al concluded after evaluating the associations between baseline and change in renal function and cardiovascular events that the presence of abnormal renal function, even with some degree of variability where there is biochemical improvement is associated with increased cardiovascular morbidity. ${ }^{11}$

For that reason, knowing initial status of electrolyte and renal function is essential for further management and or long-term outcome. Considering the paucity of the literature, the study was planned to assess clinical, laboratory especially electrolyte $\&$ renal function in acute heart failure patients during hospital admission followed by short clinical outcome. The study findings will help the physician to know the current status of these groups of patients and eventually help the patients.

\section{Methodology}

It's a prospective observational study conducted in Medicine department of Dhaka medical college hospital from 2019 July to 2019 December. All acute heart failure ${ }^{12,13}$ patients those who admitted in medicine ward for the first time were included in this study. Sampling technique was purposive, convenient. Sample size came up to 384 based on prevalence equation. ${ }^{14}$ However; we enrolled 110 patients in 6 months. Face to face interview was conducted by using a semi-structured questionnaire containing socio-demographic parameters and clinical presentations. Detailed history, clinical examinations, investigations like $\mathrm{CBC}$, Urine $\mathrm{R} / \mathrm{E}, \mathrm{C} \mathrm{S}$. Creatinine, S. 
electrolyte, like ( $\mathrm{Na}, \mathrm{K}, \mathrm{Cl}, \mathrm{HCO} 3, \mathrm{Ca} \& \mathrm{Mg}$ ), ECG, Chest X-ray, USG of renal system \& Echocardiography where needed. We assessed their clinical symptoms as a marker of improvement before hospital discharge. Data were collected in a semi structured questionnaire form along with informed consent by acting doctor who were assigned for this study. Ethical clearance was taken from ethical review committee of respective medical college. All data were analyzed by SPSS 23 version $\&$ presented as table, \& bar diagram. Measurement data of normal distribution was expressed as mean \pm standard deviation. All counts and measurement data were descriptive statistics, and the results were expressed as percentages.

\section{Result}

Total 110 patients of acute heart failure were included in this study. Mean age was 53.88 ( \pm 14.6) years. Maximum and minimum age was 82 years and 23 years respectively. Majority of the patients were aged between 51-60 years (32.0\%). See Figure-1. Among all subjects, 83 $(75.45 \%)$ were male with male-female ratio $3: 1$. Co-morbidities of patients of our study population showed that hypertension 59 (53.63\%), ischemic heart disease 42 (38.18\%), DM 39(35.45\%), chronic kidney disease 25(22.72\%), dyslipidaemia 20 (18.18\%), anaemia 19 (17.27\%), asthma/COPD 14 $(12.72 \%)$, atrial fibrillation $13(11.83 \%)$, peripartum cardiomyopathy 5 (4.54\%), SLE 4 $(3.64 \%) \&$ Tuberculosis 2 (1.8\%). (Table 1$)$.

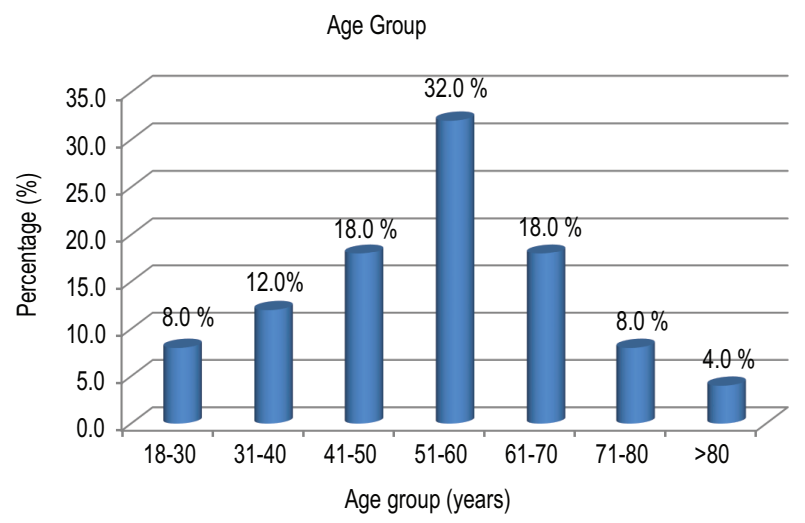

Fig.-1: Age distribution of patients with heart failure $(n=110)$
Table-I

Co-morbidities among heart failure patients $(n=110)$

\begin{tabular}{lcc}
\hline Co-morbidities & Frequency & Percentage \\
\hline Hypertension & 59 & 53.63 \\
\multicolumn{2}{l}{ Ischemic Heart Disease 42} & 38.18 \\
Diabetes mellitus & 39 & 35.45 \\
CKD & 25 & 22.72 \\
Dyslipidaemia & 20 & 18.18 \\
Anaemia & 19 & 17.27 \\
Asthma/ COPD & 14 & 12.72 \\
Atrial fibrillation & 13 & 11.81 \\
Hyperthyroid & 5 & 4.54 \\
Peripartum & 5 & 4.54 \\
cardiomyopathy & & \\
SLE & 4 & 3.64 \\
Tuberculosis & 2 & 1.81 \\
\hline
\end{tabular}

Regarding personal habits of study population, $79(72 \%)$ of total patients had history of smoking and $12(11 \%)$ were alcoholic. $45(41 \%)$ patients lead a sedentary lifestyle. All 110 (100\%) patients had shortness of breath along with palpitation 96(87.27\%), orthopnea 85(77.27\%), Paroxysmal nocturnal dyspnea 59(53.63\%), ankle edema $71(64.54 \%)$, raised JVP $75(68.18 \%)$, bilateral basal crepitation on auscultation 80(72.72\%), anaemia 19 (17.27\%) $\&$ oliguria 23(20.90\%) (Table 2). Mean duration of symptoms $13 \pm 2$ days. It is found that mean pulse rate was $85.6 \pm 8.7 \mathrm{bpm}$, mean systolic $\mathrm{BP}$ was $117.2 \pm 10 \mathrm{mmHg}$ and diastolic BP was $79.5 \pm 7.7 \mathrm{mmHg}$. Moreover, 77(70\%) were classified into Heart failure with reduced ejection faction (Table 2). Lab investigations revealed decreased Hemoglobin (10.71 \pm 1.49) $\mathrm{g} / \mathrm{dl}$, proteinuria in 31 (28.18\%), ketone body 5 $(4.5 \%)$, raised creatinine in $23(20.93 \%)$, hyponatremia 38 (34.54\%), hypernatremia 6 (5.45\%), hypokalemia 6 (5.45\%), hyperkalaemia $3(2.72 \%)$ (Table 3$)$. Regarding cardiac events $37(33.63 \%)$ encounter myocardial infarction, cardiogenic shock 29(26.36\%), Cardiac arrhythmia $27(24.5 \%)$, cardiac arrest $6(5.45 \%)$, non-cardiac events like stroke $6(5.45 \%)$, hyperosmolar hyperglycemic state $2(1.8 \%)$, diabetic ketoacidosis $5(5.45 \%)$, acute kidney injury $23(20.90 \%)$. However, among cardiac arrhythmia, Atrial fibrillation found $13(11.81 \%)$ 
followed by multiple ventricular ectopic 12 (10.90\%), ventricular tachycardia 7 (6.36\%), Complete heart block 6 (5.45\%), variable heart block 4 (3.63\%), long QT 2(1.8\%) (Table 4). Regarding short outcome 52(47.27\%) were improved, 40 (36.36\%) symptoms were persistent, $18(16.36 \%)$ expired. Cause of those deceased person were cardiogenic shock $9(50 \%)$, cardiac arrest due to Ventricular fibrillation 6 (33.33\%), DKA 2 (11.11\%) \& hemorrhagic stroke $1(5.5 \%)$ (Table 5).

Table-II

Important clinical characteristics of heart failure patients $(n=110)$

\begin{tabular}{lcc}
\hline Important Clinical signs & Frequency & Percentage \\
\hline Shortness of breath & 110 & 100 \\
Palpitation & 96 & 87.27 \\
Orthopnoea & 85 & 77.27 \\
Bilateral basal crepitation & 80 & 72.72 \\
Raised JVP & 75 & 68.18 \\
Ankle edema & 71 & 64.54 \\
Paroxysmal nocturnal & 59 & 53.63 \\
dyspnea & & \\
Atrial fibrillation & 13 & 11.81 \\
Anaemia & 16 & $14.54 \%$ \\
Oliguria & 23 & $20.91 \%$ \\
Clinical Type (based on Echo) & \\
HF with reduced & 77 & 70 \\
EFHF with preserved EF & 33 & 30 \\
\hline
\end{tabular}

Table-III

Important laboratory findings among heart failure patients during admission

\begin{tabular}{|c|c|c|}
\hline Trait & Mean \pm SD & $\begin{array}{c}\text { Frequency } \\
\text { N (\%) }\end{array}$ \\
\hline Hemoglobin & $10.71 \pm 1.49$ & \\
\hline Proteinuria (presen & & $31(28.18)$ \\
\hline Urine for ketone bo & & $5(4.5 \%)$ \\
\hline Creatinine & $1.29 \pm 0.79$ & \\
\hline RBS & $9.53 \pm 2.54$ & \\
\hline Sodium & $133.56 \pm 6.08$ & \\
\hline Hyponatremia & & $38(34.54)$ \\
\hline Hypernatremia & & $6(5.45)$ \\
\hline Potassium & $4.79 \pm 0.68$ & \\
\hline Hyperkalaemia & & $3(2.72)$ \\
\hline Hypokalaemia & & $6(5.45)$ \\
\hline $\mathrm{HCO} 3$ & $22.07 \pm 4.01$ & \\
\hline Metabolic acidosis & & 17 (15.45) \\
\hline
\end{tabular}

Table - IV

Cardiac \& Non Cardiac Events occurred during index admission of along with heart failure patients $(N=110)$

\begin{tabular}{lcc}
\hline Events & Frequency & Percentage \\
\hline Shock & 29 & 26.36 \\
Myocardial infarction & 37 & 33.63 \\
Cardiac arrhythmia & 27 & 24.5 \\
Multiple ventricular & 12 & 10.90 \\
ectopic & & \\
VT & 7 & 6.36 \\
Complete Heart Block & 6 & 5.45 \\
Variable Heart Block & 4 & 3.63 \\
Cardiac arrest & 6 & 5.45 \\
Hypokalaemia with & 2 & 1.8 \\
(Long QT) & & \\
Hyperkalaemia & 3 & 2.7 \\
Non Cardiac Events & & \\
Acute kidney injury & 23 & 20.90 \\
Stroke & 6 & 5.45 \\
Ischemic ( Cardio embolic) 5 & 4.5 \\
Hemorrhagic & 1 & 0.9 \\
Hyperglycemia & & \\
HHS & 72 & 6.41 .8 \\
DKA & 5 & 4.5 \\
\hline
\end{tabular}

Table -V

Clinical outcome among acute heart failure patients $(N=110)$

\begin{tabular}{lcc}
\hline Outcome & Frequency & Percentage \\
\hline Clinically improved & 52 & 47.27 \\
Persistent of symptoms & 40 & 36.36 \\
Death & 18 & 16.36 \\
Cardiogenic shock & 9 & 50 \\
Cardiac arrest & 6 & 33.33 \\
DKA & 2 & 11.11 \\
Hemorrhagic stroke & 1 & 5.5 \\
\hline
\end{tabular}

\section{Discussion}

Total 110 patients with acute heart failure who admitted first time in a tertiary care hospital were included. Majority of our patients were 
aged between 51-60 years (32.0\%) followed by $18 \%$ in $41-50$ years and another $18 \%$ in $61-70$ years both of which were second highest. Kabiruzzaman et al. in Bangladesh found the mean age was $54.1 \pm 15.3$ years with majority $(30.7 \%)$ patients in 51-60 years group (15). Abedin et al. also found similar result but Rahaman et al. found mean age $46 \pm 7$ years with majority $(75 \%)$ patients in 51-70 years group. ${ }^{16,17}$ Sex distribution of patients was slightly in favour of males $(75.45 \%)$ than female $(24.54 \%)$ with male female ratio $3: 1$. Kabiruzzaman et al., Abedin et al. and Araújo et al. also got similar result with male predominance. ${ }^{15,16}$ In our study, as a comorbid condition, about $54 \%$ had history of hypertension, 38\% had ischemic heart disease, $35 \%$ had DM, 18\% had dyslipidaemia, 17\% had anaemia, $13 \%$ had primary respiratory problems and rest $11.81 \%$ had history of atrial fibrillation. In the study of Rahman et al. found similar result as hypertension (45\%), diabetes $(29 \%)$, respiratory disease $(27 \%)$ and atrial Fibrillation in 9\%. ${ }^{16}$ In SOLVD (1991) clinical trial, $42 \%$ had hypertension, $26 \%$ had diabetes, $10 \%$ had Atrial Fibrillation and in MERIT-HF clinical trial, 44\% had hypertension, 25\% had diabetes and $17 \%$ had atrial fibrillation. 18.19 Regarding personal habits of study population, $72 \%$ of total patients were smoker and only $12 \%$ were alcoholic and $41 \%$ patient leads a sedentary lifestyle. Noori et al. found 55\% were current smoker among readmission population which was similar to our study. ${ }^{20}$ In this study, $100 \%$ patients presented with shortness of breath, $92 \%$ had bilateral basal crepitation on auscultation, $80 \%$ had orthopnoea, $66 \%$ had raised JVP, 64\% had bilateral ankle edema, 54\% had paroxysmal nocturnal dyspnoea and only $10 \%$ had oliguria. Rahman et al. and Barasa et al. also found same result. ${ }^{17,21}$

In the present study, mean pulse rate was $83.6 \pm 8.7 \mathrm{bpm}$, mean systolic BP was $117.2 \pm 10$ $\mathrm{mmHg}$ and diastolic BP was $79.5 \pm 7.7 \mathrm{mmHg}$. Dokainish et al. found regarding clinical characteristics, systolic blood pressure 115.2 $\pm 22 \mathrm{mmHg}$, diastolic blood pressure $76 \pm 13$ $\mathrm{mmHg}$ and heart rate $81 \pm 17 \mathrm{bpm}$ which is very much similar to us. ${ }^{22}$ Tsutsui et al. also found similar result as heart rate $70.5 \pm 12.0 \mathrm{bpm}$, systolic blood pressure $117.0 \pm 18.6 \mathrm{mmHg}$ and diastolic blood pressure $66.1 \pm 11.6 \mathrm{mmHg} .{ }^{23}$ In our study we found that, hyponatraemia was present in $34.54 \%$ of the patients while $5.45 \%$ had hypenatraemia. Barasa et al. found hyponatraemia was present in $35.6 \%$ of the patients while $7.4 \%$ had hypernatraemia. ${ }^{21}$ Hyponatraemia is particularly common in $\mathrm{CCF}$ and OPTIMIZE-HF registry recorded that 25.3\% of 47,647 heart failure patients had hyponatraemia on admission which also supports our study. ${ }^{24}$ Hyponatraemia generally identifies $\mathrm{CHF}$ patients in more advanced stages of the syndrome and, thus, a more activated neuro-hormonal system. ${ }^{25}$ Hypokalaemia in relation to hyponatraemia was less common affecting $5.45 \%$ patients followed by hyperkalaemia in $2.72 \%$ patients. Barasa et al. showed hypokalaemia and hyperkalaemia were present in $7.4 \%$ and $2.9 \%$ patients of their study. ${ }^{21}$ Hypokalemia has been associated with a poor prognosis in patients with CHF. Thus, it is likely that hypokalemia is as much a marker of other pathophysiologic factors or of the severity of CHF itself as it is an isolated predictor of adverse events. ${ }^{25}$

Frequency of acute kidney injury (AKI) in congestive cardiac failure showed $20.90 \%$ patients of $\mathrm{CCF}$ patients developed AKI. Depending on the population, $27-40 \%$ of patients hospitalized for ADHF develop acute kidney injury (AKI). ${ }^{9}$ This figure is lower than that found by Ghonemy et al. who got $47 \%$ patients with CHF had developed AKI. 26 Regarding CKD in our study population, $22.72 \%$ of total patients had chronic kidney disease (CKD). Ahmed et al. and Ezekowitz et al. also showed $37 \%$ and $39 \%$ patients with $\mathrm{CCF}$ developed chronic kidney disease respectively which is almost similar to our study. ${ }^{27,28)}$

However, among cardiac arrhythmia Atrial fibrillation found $13(11.81 \%)$ followed by multiple ventricular ectopic 12(10.90\%), ventricular tachycardia $7(6.36 \%)$, Complete heart block 6 (5.45\%), variable heart block 4 (3.63\%), long QT 2(1.8\%) Huikuri HV et al ${ }^{29} \&$ Masarone D et al. ${ }^{30}$ found almost similar but Lip GYH et al. ${ }^{31}$ found same arrhythmia but percentage were different. Regarding short 
clinical outcome we found 52(47.27\%) were improved, 40 (36.36\%) symptoms were persistent, 18 (16.36\%) expired, Cause of those deceased person were cardiogenic shock 9(50\%), cardiac arrest due to Ventricular fibrillation 6 (33.33\%), DKA 2 (11.11\%) \& hemorrhagic stroke $1(5.5 \%)$. A systematic death review found that overall, $62 \%$ of underlying causes of death were cardiovascular, with a large proportion $(25 \%)$ of underlying causes attributable to CHD. Progressive pump failure was the major nonCHD cause of cardiovascular death $(16 \%$ of all underlying causes). Respiratory disease (infectious and noninfectious) was the leading underlying cause of noncardiovascular death (10\%), followed by cancer (9\%). ${ }^{32}$

Our study has some limitations like small sample size, prospective observational but for short duration. It will be better if it was prospective longitudinal cohort.

\section{Conclusion:}

Clinical characteristics of HF are more or less similar but those who have reduced ejection fraction with comorbidities are very prone to develop cardiac arrhythmia. It might be due to structural change or electrolyte imbalance. We should always consider diabetes, stroke, renal impairment and cardiac arrhythmias as risk factor for increased mortality among heart failure patients. Nevertheless, this small study is an effort to describe clinical presentation of acute heart failure with short clinical outcome, large scale study needed to find out exact situation of heart failure in Bangladesh.

\section{References}

1. van Riet EE. Epidemiology of heart failure: the prevalence of heart failure and ventricular dysfunction in older adults over time. A systematic review. Eur. J. Heart Fail. 2016; 18: 242-52.

2. Schocken DD. Prevention of heart failure: a scientific statement from the American Heart Association Councils on Epidemiology and Prevention, Clinical Cardiology, Cardiovascular Nursing, and High Blood Pressure Research; Quality of Care and Outcomes Research Interdisciplinary Working Group; and Functional Genomics and Translational Biology Interdisciplinary Working Group. Circulation.2008; 117: 2544-65.

3. Redfield MM. Burden of systolic and diastolic ventricular dysfunction in the community: appreciating the scope of the heart failure epidemic. JAMA. 2003; 289: 194-202.

4. Urso C, Brucculeri S, Caimi G. Acid-base and electrolyte abnormalities in heart failure: pathophysiology and implications. Heart Fail Rev. 2015;20(4):493-503.

5. Weglicki W, Quamme G, Tucker K, Haigney M RL. Potassium, magnesium, and electrolyte imbalance and complications in disease management. ClinExpHypertens. 2005;1:95-112.

6. Resnick LM, Barbagallo M, Dominguez LJ, Veniero JM, Nicholson JP, Gupta RK. Relation of cellular potassium to other mineral ions in hypertension and diabetes. Hypertension. 2001; 38(3 Pt 2):709-12.

7. Udani SM, Koyner JL. The Effects of Heart Failure on Renal Function. Cardiol Clin. 2010;28(3):453-65.

8. Bagshaw SM. Epidemiology of cardio-renal syndromes: workgroup statements from the 7th ADQI Consensus Conference. Nephrol. Dial. Transplant. 2010;25: 1406-16.

9. House AA. Definition and classification of cardio-renal syndromes: workgroup statements from the 7th ADQI Consensus Conference. Nephrol. Dial. Transplant.2010; 25: 1416-20.

10. Khan NA, Ma I, Thompson CR, Humphries K, Salem DN, Sarnak MJ, et al. Kidney Function and Mortality among Patients with Left Ventricular Systolic Dysfunction. J Am Soc Nephrol. 2006;17(1):244-53.

11. Weiner DE, Krassilnikova M, Hocine T, et al. CKD classification based on estimated GFR over three years and subsequent cardiac and mortality outcomes: a cohort study. BMC Nephrology. 2009;10:26- 37 .

12. Hunt SA, Abraham WT, Chin MH, Feldman AM, Francis GS, Ganiats TG, et al. ACC/AHA 2005 Guideline Update for the Diagnosis and Management of Chronic Heart Failure in the Adult. Circulation. 2005; 112(12):e154-235.

13. NICe clinical guideline for diagnosis and management of heart failure in primary and secondary care. September 2018.

14. Pourhoseingholi MA, Vahedi M, Rahimzadeh M. Sample size calculation in medical studies. Gastroenterol Hepatol Bed Bench. 2013;6(1):14-17.

15. Kabiruzzaman M, Malik F, Ahmed N, Badiuzzaman M, Choudhury S, Haque T, et al. Burden of Heart Failure Patients in a Tertiary Level Cardiac Hospital. J Bangladesh Coll Physicians Surg. 2010; 28 (1):289.

16. Rahman MT, Majumder A, Rahman A, Chowdhury AW. Clinical Presentation of Heart Failure Patients Admitted in National Institute of Clinical Presentation 
of Heart Failure Patients Admitted in National Institute of Cardiovascular Diseases, Dhaka. 2014; (September 2015).

17. Abedin MZ, Banerjee SK, Hoque H, Mahmood M, Krishna D, Samsuzzaman SM, et al. Demographic and Socio-Eeconomic Characteristics of Patients with Congestive Heart failure Presenting to the Cardiac Emergency of Bangabandhu Sheikh Mujib Medical University. Univ Hear J. 2016; 12(2):76-81.

18. SOLVD Investigators, Yusuf S, Pitt B, Davis CE, Hood WB, Cohn JN. Effect of enalapril on survival in patients with reduced left ventricular ejection fractions and congestive heart failure. N Engl J Med. 1991;325(5):293-302.

19. MERIT Investigators. Effect of metoprolol CR/XL in chronic heart failure: Metoprolol CR/XL Randomised Intervention Trial in Congestive Heart Failure (MERIT$\mathrm{HF}$ ). Lancet (London, England). 1999 Jun 12; 353(9169):2001-7.

20. Noori A, Shokoohi M, Baneshi MR, Naderi N, Bakhshandeh H, Haghdoost AA. Impact of socioeconomic status on the hospital readmission of Congestive Heart Failure patients: a prospective cohort study. Int $\mathrm{J}$ Heal Policy Manag. 2014; 3 (5):251-7.

21. Barasa FA. Socio-demographics, Clinical profile and Disposition of In-patients with Acute Decompensated Heart Failure at Kenyatta National Hospital. Dissertation published in Library of Univesity of Nairobi.

22. Dokainish H, Teo K, Zhu J, Roy A, Al-Habib K, ElSayed A, et al. Heart failure in low- and middleincome countries: Background, rationale, and design of the INTERnational Congestive Heart Failure Study (INTER-CHF). Am Heart J. 2015;170(4):627-634.e1.

23. Tsutsui H, Rn MT. Clinical characteristics and outcomes of heart failure with preserved ejection fraction/: Lessons from epidemiological studies. 2010
24. Abraham WT. Managing Hyponatraemia in Heart Failure. Congest Hear Fail. 2008; (1):57:39-41.

25. Dei Cas L, Metra M, Leier C V. Electrolyte disturbances in chronic heart failure: Metabolic and clinical aspects. Clin Cardiol. 1995 Jul;18(7):370-6.

26. Ghonemy TA, Allam HM, Elokely AM, Lotfy E, Elnahal $\mathrm{S}$, Gharib AF. Prevalence of acute kidney injury in cardiac patients in the Intensive Care Unit. 2016; 60-5.

27. Beta-Blocker Evaluation of Survival Trial Investigators, Eichhorn EJ, Domanski MJ, KrauseSteinrauf H, Bristow MR, Lavori PW. A trial of the beta-blocker bucindolol in patients with advanced chronic heart failure. N Engl J Med. 2001;344(22):1659-67.

28. Ezekowitz J, Bc H, Ms C, Mcalister FA, Ms C, Humphries KH, et al. Renal Insufficiency and Heart Failure The Association Among Renal Insufficiency, Pharmacotherapy, and Outcomes in 427 Patients With Heart Failure and Coronary Artery Disease. J Am Coll Cardiol. 2004;44(8):1587-92.

29. Huikuri HV et al. Sudden death due to cardiac arrhythmias. N Engl J Med. 2001; 345(20):1473-82.

30. Masarone D et al. Management of arrhythmias in heart failure. J Cardiovasc Dev Dis. 2017; 4 (1):3.

31. Lip GYH et al. European Heart Rhythm Association/ Heart Failure Association joint consensus document on arrhythmias in heart failure, endorsed by the Heart Rhythm Society and the Asia Pacific Heart Rhythm Society. EP Europace. 2016;18(1):12-36

32. Lee DS, Gona P, Albano I, et al. A systematic assessment of causes of death after heart failure onset in the community: impact of age at death, time period, and left ventricular systolic dysfunction. CircHeart Fail. 2011;4(1):36-43. doi:10.1161/Circheartfailure. 110.957480 